\title{
MANEJO SUSTENTADO PARA FLORESTA DE VÁRZEA NA AMAZÔNIA ORIENTAL ${ }^{1}$
}

João Ricardo Vasconcellos Gama², Michelliny de Matos Bentes-Gama ${ }^{3}$ e José Roberto Soares Scolforo ${ }^{4}$

\begin{abstract}
RESUMO - Este estudo teve como objetivo definir opções de colheita em bases sustentadas para florestas de várzea no estuário amazônico. Os dados foram coletados na propriedade florestal da Exportadora de Madeira do Pará Ltda. (Emapa), localizada no Município de Afuá, ao norte do Estado do Pará. A amostragem foi realizada em 29 parcelas de $5.000 \mathrm{~m}^{2}$. Foram medidas todas as árvores e palmeiras com dap $\geq 45 \mathrm{~cm}$. As espécies comerciais que apresentaram condições de serem colhidas por terem apresentado os melhores índices fitossociológicos e qualitativos foram: Virola surinamensis, Carapa guianensis e Hymenaea oblongifolia. Entre as espécies potenciais, destacou-se Terminalia dichotoma; e no grupo das espécies não-comerciais, Eschweilera coriacea, Swartizia racemosa e Licania macrophylla. Os resultados indicaram que a floresta pode ser manejada, adotando-se o plano de colheita que utiliza um Quociente de De Liocourt $50 \%$ maior do que o original $(q=2,61)$ e remoção de $30 \%$ da área basal, o que corresponde a um lucro potencial de US\$ 3.945,40/ha.
\end{abstract}

Palavras-chave: Fitossociologia, ecologia florestal e Manejo florestal.

\section{SUSTAINABLE MANAGEMENT FOR FLOODPLAIN FOREST IN EASTERN AMAZONIA}

\begin{abstract}
The aim of this study was to define tree cut options in a sustainable basis for floodplain forests in the Amazonian Estuary. Data were obtained from the survey carried out in the forest lands of Wood Exports of Pará Ltda. - EMAPA Enterprise, located in Afuá County, North of Pará State. Sampling was performed in 29 plots of $5.000 \mathrm{~m}^{2}$. There were surveyed all trees, including palm trees with diameter at $1.30 \mathrm{~m}$ above ground level $(\mathrm{dbh}) \geq 45 \mathrm{~cm}$. Trade species with possibility to be harvested for presenting best phytossociological and qualitative indexes were: Virola surinamensis, Carapa guianensis and Hymenaea oblongifolia; among potential species Terminalia dichotoma was chosen; while in the non-trade species group, Eschweilera coriacea, Swartizia racemosa and Licania macrophylla were selected. Results showed that the forest management can be done by adopting a harvesting plan with a De Liocourt value 50\% higher than the original $(q=2,61)$ one and removal of $30 \%$ of basal area, which corresponds to a potential income of US\$3.945,40/ha.
\end{abstract}

Key-words: Phytossociology, forest ecology and forest management.

\footnotetext{
${ }^{1}$ Recebido em 16.02.2004 e aceito para publicação em 10.08.2005.

${ }^{2}$ Gama Florestal, Trav. Timbó no 1520 Pedreira, CEP 66085-654 Belém-PA. Email: <jrvgama@uol.com.br>

${ }^{3}$ Embrapa Rondônia, BR 364, km 5,5, Caixa Postal 406, CEP78900-970 Porto Velho-RO. E-mail: <mbgama@cpafro.embrapa.br>

${ }^{4}$ Departamento de Ciências Florestais da UFLA, Caixa Postal 37, CEP 37200-000 Lavras-MG. E-mail: <scolforo@ ufla.br>
} 


\section{INTRODUÇÃO}

A garantia de uma produção contínua de madeira, associada à conservação da biodiversidade de florestas nativas como a Amazônia, pode ser alcançada mediante o manejo florestal sustentável (MFS). A finalidade do manejo florestal é conseguir que as florestas forneçam continuamente benefícios econômicos, ecológicos e sociais, mediante um planejamento mínimo para o aproveitamento dos recursos madeireiros e nãomadeireiros disponíveis.

As evidências dos benefícios do manejo florestal para garantir a conservação da biodiversidade de florestas nativas e o bem-estar das populações que delas dependem são relatadas por Buschbacher (1990), Finegan et al. (1993) e Bertault et al. (1995), entre outros. Porém, grande parte dos estudos realizados na Amazônia é direcionados às florestas de terra firme (SILVA, 1989; UHL e VIEIRA, 1989; SILVAet al., 1995; BARRETO et al., 1998; MINETTI et al., 2000; VIDAL et al., 2002).

As florestas de várzea, o segundo maior alvo de exploração predatória na Amazônia, são pouco estudadas com relação aos critérios de colheita florestal, podendose citar os trabalhos de Macedo (1996) e Ramos (2000).

As florestas de várzea correspondem à segunda maior formação vegetal da bacia amazônica, ocupando uma superfície de cerca de $75.880,8 \mathrm{~km}^{2}$ (ARAÚJO et al., 1986). Apesar do grande potencial das várzeas para a agricultura, principalmente culturas de ciclo curto, o aproveitamento florestal se apresenta como uma das atividades mais importantes, pois ainda existem muitas áreas com alto estoque de produtos madeireiros e nãomadeireiros (RIBEIRO et al., 2004).

Diante do exposto e devido à carência de estudos sobre o tema em florestas de várzea, o objetivo deste estudo foi definir opções de colheita em bases sustentáveis para florestas de várzea no estuário amazônico.

\section{MATERIAL E MÉTODOS}

\subsection{Descrição da Área}

A área de estudo fica na propriedade florestal da Exportadora de Madeiras do Pará Ltda. (EMAPA), localizada no Rio Santana, Município de Afuá, ao norte do Pará $\left(0^{\circ} 09^{\prime} 32^{\prime \prime S}\right.$ e $\left.50^{\circ} 23^{\prime} 31^{\prime \prime} \mathrm{W}\right)$. O clima da região é quente e úmido, do tipo Ami, segundo Köeppen;

R. Árvore, Viçosa-MG, v.29, n.5, p.719-729, 2005 a precipitação média anual é de $2.500 \mathrm{~mm}$ e a temperatura média anual, de $26^{\circ} \mathrm{C}$ (SUDAM, 1984). A topografia da região é plana a suavemente ondulada, com predominância de solos hidromórficos, variando de húmicos a pouco húmicos (VIEIRA, 1988).

\subsection{Amostragem e Coleta de Dados}

A partir do mapa da área e com base nas recomendações da FAO (1974), foram distribuídas sistematicamente 29 unidades amostrais de $20 \mathrm{~m} \times 250 \mathrm{~m}$, em uma unidade de trabalho (UT) de 60 ha. Em cada parcela foram mensuradas todas as espécies arbóreas e palmeiras com diâmetro à altura de 1,30 $\mathrm{m}$ do solo (dap) $\geq 45 \mathrm{~cm}$ e anotados o nome regional; altura total e comercial; qualidade de fuste: reto, pouco tortuoso e tortuoso; presença de cipó: ausência de cipó, cipó no fuste, cipó na copa e cipó na árvore toda; classe de iluminação: luz total na copa, luz parcial na copa e luz indireta na copa; e raízes escoras: ausência e presença.

Foi contabilizado o número de toras de $4 \mathrm{~m}$ de cada árvore, classificadas de acordo com os padrões de comercialização local em: tora de $1^{\mathrm{a}}\left(\mathrm{T}_{1}\right)$ - circunferência no meio da tora com casca: $\mathrm{C}^{3} 150 \mathrm{~cm}$; tora de $2^{\mathrm{a}}\left(\mathrm{T}_{2}\right)$ : $130 \mathrm{~cm} \leq \mathrm{C}<150 \mathrm{~cm}$; e tora de $3^{\mathrm{a}}\left(\mathrm{T}_{3}\right)$ : $110 \mathrm{~cm} \leq \mathrm{C}<130 \mathrm{~cm}$. Contabilizou-se o número de indivíduos de Euterpe oleracea Mart. com dap $\geq 10 \mathrm{~cm}$, que foram classificados em palmito de $1^{\mathrm{a}}$ (dap $\left.\geq 15 \mathrm{~cm}\right)$ e palmito de $2^{\mathrm{a}}(10 \mathrm{~cm} \leq$ dap $<15 \mathrm{~cm})$.

Adotou-se também a classificação local das espécies, a saber: comerciais - espécies comercializadas pela empresa; potenciais - espécies comercializadas pelas pequenas serrarias da região e não-comerciais - ainda não comercializadas, mas utilizadas pelos ribeirinhos. As espécies foram identificadas por especialistas do Laboratório de Botânica da Embrapa Amazônia Oriental, em Belém, PA. Adotou-se o sistema de classificação de Cronquist (1988).

\subsection{Análise dos Dados}

\subsubsection{Definição dos Critérios de Colheita}

Como critério de colheita, levaram-se em consideração: (1) número de árvores por espécie e por classe diamétrica; (2) utilização de uma equação volumétrica para árvores com dap $\geq 45 \mathrm{~cm}$ : $\ln \mathrm{V}=-7,62812$ $+2,18090 \operatorname{lnD}\left(\mathrm{R}^{2}=0,836\right.$ e $\left.\mathrm{CV}_{\%}=16,0\right)$, em que $\mathrm{V}=$ volume comercial com casca e $\mathrm{D}=$ diâmetro à altura 
de 1,30 m do solo, ou imediatamente acima da sapopema (SILVA et al., 1984); (3) índices fitossociológicos que caracterizam a estrutura da floresta (CURTIS e MCINTOSH, 1951; FINOL, 1971; JARDIM e HOSOKAWA, 1986/1987), não se considerou a regeneração natural, uma vez que o nível de inclusão adotado não contemplou a avaliação desse componente da fitocenose; e (4) conceito de floresta balanceada, em que o modelo adotado foi o de Meyer linearizado: $\ln Y_{i}=\ln \beta_{0}+\beta_{1} X_{i}+\varepsilon_{i}$; em que $Y_{\mathrm{i}}=$ número de árvores da i-ésima classe de diâmetro; $X_{\mathrm{i}}=$ valor central da i-ésima classe de diâmetro; $\ln =$ logaritmo neperiano; $\beta_{0}$ e $\beta_{1}=$ coeficientes a serem estimados; e $\varepsilon_{\mathrm{i}}$ : erro de estimativa (CAMPOS et al., 1983).

A princípio, a floresta foi balanceada considerando valores de Quociente de De Liocourt - q ( 'q' original, $50 \%$ maior e $100 \%$ maior) e área basal da floresta remanescente $-\mathrm{G}_{\mathrm{rem}}$ (porcentuais de remoção: 10, 20, $25,30,40$ e $50 \%$ ), optou-se por não promover qualquer alteração no diâmetro máximo. Portanto, foram simuladas 18 opções de remoção de árvores por classe diamétrica. Posteriormente, para uma espécie ter o seu número de indivíduos removido por classe de diâmetro, considerou-se como restrições: (1) apresentar densidade relativa $\geq 1 \%$ e, ou, dominância relativa $\geq 1 \%$; (2) nãoremoção das espécies proibidas de corte (BRASIL, 1995), nesse caso apenas Hevea brasiliensis (Willd. ex Juss.) M. Arg., e as mortas em pé; (3) diâmetro mínimo de corte $\geq 45 \mathrm{~cm}$; (4) e, entre as espécies possíveis de serem removidas, o mínimo de $10 \%$ dos indivíduos foi mantido - por classe diamétrica - como medida para garantir a permanência dos porta-sementes.

A determinação do número de árvores por espécie, a ser removido por classe de diâmetro para os cenários de manejo, foi obtida por meio da expressão desenvolvida por Scolforo (1997): $N R=\left\{N E_{i} D_{i} /\left[\left(\sum_{i=1}^{s} N E_{i} D_{i}\right)-\left(\sum_{i=1}^{s} N E D R D o R_{i} D_{i}\right)\right]\right\} \cdot F_{r}$, em que: $\mathrm{NR}=$ número de indivíduos a serem removidos por espécie e por classe de diâmetro; $\mathrm{NE}_{\mathrm{i}} \mathrm{D}_{\mathrm{i}}=$ número de indivíduos $(\mathrm{N})$ da i-ésima espécie $\left(\mathrm{E}_{\mathrm{i}}\right)$ na i-ésima classe diamétrica $\left(\mathrm{D}_{\mathrm{i}}\right) ; \mathrm{NEDRDoRD}_{\mathrm{i}}=$ número de indivíduos $(\mathrm{N})$ das espécies $\left(\mathrm{E}_{\mathrm{i}}\right)$ com densidade relativa (DR) $<1$ (raras), e, ou, dominância relativa $($ DoR $)<$ 1, na i-ésima classe diamétrica $\left(\mathrm{D}_{\mathrm{i}}\right)$; e $\mathrm{Fr}=$ número de indivíduos a serem removidos na i-ésima classe de diâmetro, conforme o conceito de floresta balanceada.

\subsubsection{Cenários de Manejo}

Dentre as propostas de colheita para a floresta, foram considerados os cenários: (1) colheita das espécies listadas como comerciais; (2) colheita das espécies listadas como comerciais e potenciais; e (3) colheita das espécies listadas como comerciais, potenciais e não-comerciais.

Para o processamento dos dados foi utilizado o "software" SISNAT - Sistema de Manejo para Florestas Nativas - desenvolvido por Scolforo (1998).

\subsubsection{Valoração dos Planos de Colheita}

Para a valoração do potencial produtivo de madeira em pé foi utilizado o preço regional - obtido por meio de entrevistas - por classe de tora das espécies comerciais. Já os preços das classes de tora das espécies potenciais e não-comerciais foram definidos por meio da média dos três menores preços por classes de toras (Quadro 1), obtida por intermédio das seguintes expressões: $R P M_{i}=\left(P_{T 1} \cdot N T_{T 1}\right)+\left(P_{T 2} \cdot N T_{T 2}\right)+\left(P_{T 3} \cdot N T_{T 3}\right)$ e $R P T=\sum_{i=1}^{S} R P M_{i}$, em que: $\mathrm{RPM}_{\mathrm{i}}=$ receita potencial de madeira em tora da i-ésima espécie, em US\$; $\mathrm{P}_{\mathrm{Ti}}=$ preço médio por classe de tora da i-ésima espécie, em US\$; $\mathrm{NT}_{\mathrm{Ti}}=$ número de toras da i-ésima espécie, para a j-ésima classe de tora; RPT = receita potencial de toras, em US\$; e s = número de espécies. O comprimento médio das toras comercializadas na região de estudo é de $4 \mathrm{~m}$.

Quadro 1 - Preço médio da tora (PMT), por classe de tora (T), das espécies madeireiras comercializadas pela Emapa, no Município de Afuá, Pará

Table 1 - Average price of bole (PMT), by bole class $(T)$, of woody species traded by Emapa in Afuá, Pará

\begin{tabular}{lccc}
\hline \multirow{2}{*}{ Espécie } & \multicolumn{3}{c}{ PMT (US\$) } \\
\cline { 2 - 4 } & $\mathrm{T}_{1}$ & $\mathrm{~T}_{2}$ & $\mathrm{~T}_{3}$ \\
\hline Comerciais & & & \\
Bombax munguba Mart. & 8,33 & 4,44 & 0,00 \\
Carapa guianensis Aubbl. & 16,67 & 8,33 & 5,00 \\
Cedrela odorata Ruiz \& Pav. & 22,22 & 13,89 & 8,33 \\
Hymenaea oblongifolia Huber & 17,78 & 10,56 & 6,11 \\
Jacaranda copaia (Aubl) D. Don. & 11,11 & 6,67 & 4,44 \\
Simaruba amara Aubl. & 11,11 & 6,67 & 4,44 \\
Virola sp. & 16,67 & 8,33 & 5,00 \\
Virola surinamensis (Rol) Warb. & 16,67 & 8,33 & 5,00 \\
Vochysia máxima Ducke & 11,11 & 6,67 & 4,44 \\
Potenciais & 10,18 & 5,93 & 2,96 \\
Não-comerciais & 10,18 & 5,93 & 2,96 \\
\hline
\end{tabular}

Em que: $\mathrm{T}_{1}=$ tora de $1^{\mathrm{a}}-\mathrm{C} \geq 150 \mathrm{~cm} ; \mathrm{T}_{2}=$ tora de $2^{\mathrm{a}}-130$ $\mathrm{cm} \leq \mathrm{C}<150 \mathrm{~cm} ; \mathrm{T}_{3}=$ tora de $3^{\mathrm{a}}-110 \mathrm{~cm} \leq \mathrm{C}<130 \mathrm{~cm} ; \mathrm{e}$ $\mathrm{C}=$ circunferência no meio da tora.

Where: $T_{1}=1^{\text {st }}$ class bole $-C \geq 150 \mathrm{~cm} ; T_{2}=2^{\text {nd }}$ class bole -130 $\mathrm{cm} \leq C<150 \mathrm{~cm} ; T_{3}=3^{\text {rd }}$ class bole $-110 \mathrm{~cm} \leq C<130 \mathrm{~cm}$; and $C=$ circumference in the middle of the bole.

R. Árvore, Viçosa-MG, v.29, n.5, p.719-729, 2005 
Na valoração do estoque de palmito, da palmeira Euterpe oleracea, foram considerados os indivíduos fornecedores de palmito com as características desejáveis para a comercialização local e o preço por classe de palmito (palmito de $1^{\mathrm{a}}$ - US $\$ 0,17$ e palmito de $2^{\mathrm{a}}$ - US\$ 0,11 ), utilizando-se as seguintes expressões: $R P_{i}=P_{C P i} \cdot N P_{C P i}$ e $R P P=\sum_{i=1}^{C P} R P_{i}$, em que: $\mathrm{RP}_{\mathrm{i}}=$ receita potencial da i-ésima classe de palmito, em US\$; $\mathrm{P}_{\mathrm{CPi}}=$ preço da i-ésima classe de palmito, em US\$; $\mathrm{NP}_{\mathrm{CPi}}=$ número de plantas na i-ésima classe de palmito; $\mathrm{CP}=$ número de classes de palmitos utilizadas; e RPP = receita potencial de palmitos, em US\$.

A receita potencial final do plano de colheita foi obtida mediante a expressão: $\mathrm{RPF}=\mathrm{RPT}+\mathrm{RPP}, \mathrm{em}$ que: $R P F=$ receita potencial final, em US\$; e RPT e RPP, já definidos anteriormente.

\subsubsection{Valoração da Madeira Processada}

Para a valoração da madeira processada advinda das prescrições de manejo, foi calculado por espécie, nas diferentes classes de tora $(\mathrm{T})$, o volume francon $\left(\mathrm{V}_{\text {francon }}\right)$ e o volume de madeira serrada $\left(\mathrm{VMS}_{\mathrm{i}}\right)$, da seguinte maneira: $V M S_{i}=V_{\text {francon }} . N M T \cdot N_{\exp .} f c$, em que: $\mathrm{V}_{\text {francon }}=\left(\frac{\overline{\mathrm{C}}_{\mathrm{T}_{\mathrm{j}}}}{4}\right)^{2} . \mathrm{L} ; \bar{C}_{T_{j}}=$ circunferência média, sem casca, da j-ésima classe de tora $\left(\mathrm{T}_{1}=2,00 \mathrm{~m}, \mathrm{~T}_{2}=1,40 \mathrm{~m}\right.$ $\left.\mathrm{e} \mathrm{T}_{3}=1,20 \mathrm{~m}\right) ; \mathrm{L}=$ comprimento da tora $(4 \mathrm{~m}) ; \mathrm{NMT}=$ número médio de toras da j-ésima classe de tora por árvore; $\mathrm{N}_{\text {exp. }}=$ número de indivíduos removidos da $\mathrm{i}$ ésima espécie por hectare; e fc = fator de correção de 0,72 para volume de madeira serrada, o que resulta no aproveitamento da Emapa, que é de 57\%. Conforme Barros et al. (2002), essa taxa de aproveitamento pode chegar a $58,2 \%$.

Para o cálculo da receita da atividade foi usado o produto do volume total de madeira serrada (VTMS) com a estimativa do preço de comercialização (EPC). Esses preços foram: US $\$ 400,00 / \mathrm{m}^{3}$ para as espécies comerciais; US $\$ 250 / \mathrm{m}^{3}$ para as espécies potenciais; e US\$ $100 / \mathrm{m}^{3}$ para as espécies não-comerciais. Houve cotação de preço para as espécies não-comerciais com a finalidade de verificar o impacto financeiro da inclusão de espécies que apresentaram estoque para comercialização, mas que ainda não estão incluídas no mercado. Os preços foram estimados pela Emapa em janeiro de 1999; o dólar na época estava cotado em $\mathrm{R} \$ 1,80$.

R. Árvore, Viçosa-MG, v.29, n.5, p.719-729, 2005
O lucro potencial oriundo do plano de manejo foi obtido pela seguinte expressão: $L P=V T M S . E P C . E L$, em que: $\mathrm{LP}=$ lucro potencial (US\$/ha), VTMS = volume total de madeira serrada da i-ésima espécie $\left(\mathrm{m}^{3} / \mathrm{ha}\right)$, EPC = estimativa do preço de comercialização (US\$/ $\mathrm{m}^{3}$ ) e EL = estimativa de lucro $-60 \%$ do preço de venda da madeira serrada (margem média de lucro praticada pela Emapa).

\section{RESULTADOS E DISCUSSÃO}

\subsection{Análise Estrutural}

Foram amostradas 44 espécies arbóreas com dap $\geq 45 \mathrm{~cm}$, entre as quais seis comerciais, 10 potenciais e 28 não-comerciais. As que apresentaram os maiores valores de importância foram: Virola surinamensis, Eschweilera coriacea Mart. ex Berg. e Licania macrophylla Benth. Com relação às comerciais, a densidade absoluta demonstrou que Virola surinamensis, Carapa guianensis e Hymenaea oblongifolia apresentaram condições de serem aproveitadas, pois essas espécies tornaram disponíveis 11,17 ind./ha. Essas espécies também apresentaram alta dominância relativa (DoR) e os maiores valores de importância, ampliados economicamente (IVIAE)

Virola surinamensis apresentou $97,79 \%$ das árvores com fuste retilíneo. Dessas, $60,9 \%$ das toras com $4 \mathrm{~m}$ de comprimento classificadas como $\mathrm{T}_{1}$ - circunferência no meio da tora $(\mathrm{C}) \geq 150 \mathrm{~cm} ; 24,1 \%$ do tipo $\mathrm{T}_{2}$ $(130 \mathrm{~cm} \leq \mathrm{C}<150 \mathrm{~cm})$; e os $15 \%$ restantes ocorreram na classe $\mathrm{T}_{3}(110 \mathrm{~cm} \leq \mathrm{C}<130 \mathrm{~cm})$. Os $2,21 \%$ dos fustes restantes apresentaram pouca tortuosidade.

Carapa guianensis teve $92,48 \%$ das árvores com fuste retilíneo. Dessas, 73,2\% das toras eram do tipo $\mathrm{T}_{1} ; 20,3 \%$ do $\mathrm{T}_{2}$; e os $6,5 \%$ restantes, classificadas como $\mathrm{T}_{3}$. Os demais $7,52 \%$ apresentaram fuste com pouca tortuosidade.

Hymenaea oblongifolia apresentou 96,62\% das árvores com fuste retilíneo. Dessas, $62,0 \%$ das toras eram do tipo $\mathrm{T}_{1} ; 24,3 \%$ foram classificadas como $\mathrm{T}_{2}$; e os $13,7 \%$ restantes, como $\mathrm{T}_{3}$. Os demais $3,38 \%$ exibiram fustes com pouca tortuosidade.

Entre as espécies potenciais, destacaram-se Terminalia dichotoma G.F.W. Mey, utilizada na produção de tábuas e lambril, entre outras peças para construções de casas e Platymiscium trinitati Benth., cuja madeira é de ótima qualidade para a fabricação de móveis, 
totalizando 1,1 árvore por hectare. Se a exploração florestal fosse realizada agregando ao plano de colheita as espécies potenciais, o número de árvores disponíveis teria um acréscimo de apenas $9,85 \%$ em relação às espécies comerciais.

Entre as espécies não-comerciais, destacaram-se Eschweilera coriacea, Licania macrophylla, Swartizia racemosa Benth. e Parinari excelsa Sabine. São espécies utilizadas pelos ribeirinhos na construção de casas e barcos, mas que, devido à falta de expansão de mercado, não são comercializadas em larga escala na região. Essas espécies representaram uma densidade de 16,96 ind./ ha, com $88,2 \%$ classificadas como tendo fuste retilíneo. Dessas, $65,6 \%$ tinham toras do tipo $\mathrm{T}_{1}, 23,6 \%$ foram classificadas como $\mathrm{T}_{2}$ e $10,8 \%$, como $\mathrm{T}_{3}$. Os demais $11,8 \%$ apresentaram fustes com pouca tortuosidade.

Se a execução de uma exploração florestal com o objetivo de maximização da produção de madeira fosse realizada, incluindo também as espécies até então classificadas como não-comerciais, o número de árvores disponíveis para o beneficiamento pela madeireira seria de 29,23 ind./ha, o que corresponderia ao aumento de $161,7 \%$ em relação às espécies que atualmente apresentam demanda de comercialização no mercado nacional e internacional de madeiras tropicais, como Virola surinamensis, Carapa guianensis e Hymenaea oblongifolia.

\subsection{Critérios de Colheita}

Das 18 opções de remoção de árvores inicialmente testadas, apenas oito apresentaram condições de serem utilizadas por não exibirem déficit de árvores em nenhuma classe de diâmetro. Verificou-se que a manutenção da estrutura original das classes de diâmetro, "q" original $(q=1,74)$, causou déficit de árvores em todas as combinações consideradas. Vale destacar que o "q" original foi calculado a partir da distribuição diamétrica estimada pelo modelo de Meyer.

Quando o valor do “q” original foi aumentado em 50\% ( $(q=2,61)$, o déficit de árvores só ocorreu na remoção de $10 \%$ da área basal. Para o quociente de De Liocourt aumentado em $100 \%(q=3,48)$, ou o dobro do "q" original, o déficit de árvores foi identificado para a intensidade de remoção de 10, 20 e 25\% da área basal.

Dentre as opções avaliadas, somente aquelas em que o quociente (q) de De Liocourt foi superior ao
" $\mathrm{q}$ " original se mostraram aptas a contribuir para a definição dos critérios de colheita. Esse fato expressa que, para a área basal remanescente estabelecida, é removido, proporcionalmente, maior número de árvores nas maiores classes diamétricas. Isso é desejável do ponto de vista de manejo, já que são as árvores que atingiram a maturidade e, ou, estão ultrapassando-a, rumo à senescência, as que serão exploradas. Desse modo, extraem-se da floresta indivíduos de maior dimensão sem, com isso, comprometer os conceitos de produção sustentável.

Quando o quociente de De Liocourt foi igual a 2,61 e a remoção da área basal $\left(\mathrm{G}_{\mathrm{rem}}\right)$ na intensidade de $20 \%$, a prescrição foi a colheita de 4,89 ind./ha com volume do fuste de $28,57 \mathrm{~m}^{3} / \mathrm{ha}$. Quando as remoções foram de 25, 30, 40 e 50\% da área basal, o número de árvores a serem colhidas foi de 6,$9 ; 8,9 ; 12,92 ;$ e 16,94 ind./ha, respectivamente, e seus volumes foram de 35,24 ; 41,$91 ; 55,24$; e $68,58 \mathrm{~m}^{3} / \mathrm{ha}$.

Considerando a sugestão de Silva (1989) de que a exploração nas florestas de terra firme deve estar situada entre 20 e $40 \mathrm{~m}^{3} /$ ha e que a produtividade do local é um fator que influencia a intensidade da exploração, acredita-se que os planos que contemplam a remoção de 40 e $50 \%$ da área basal não sejam considerados.

Quando o "q" foi igual a 3,48 e a remoção em área basal, 30, 40 e 50\%, o número de árvores a serem colhidas por hectare foi 6,$21 ; 10,61$; e 15,02 , respectivamente. Os volumes nesse caso foram de 42,83; 53,06; e 69,24 $\mathrm{m}^{3} / \mathrm{ha}$. Adotando a mesma sugestão de Silva (1989), recomenda-se não implementar os planos que compreendam a remoção de 40 e $50 \%$ da área basal.

Os resultados também indicaram que foi possível adotar qualquer um dos seguintes planos: opções de $q=2,61$, com remoção de 20 e $25 \%$ da área basal; e as opções de $q=2,61$ e q = 3,48, com remoção de $30 \%$ da área basal. Neste trabalho foi adotada a opção de colheita com q $=2,61$ e remoção de $30 \%$ da área basal. Vale ressaltar que, para a floresta remanescente ficar balanceada, é necessário que o cenário de manejo obdeça às indicações de colheita por classe de diâmetro (Quadro 2).

Ainda foi incluída, em cada opção de colheita, a mesma produção não-madeireira de Euterpe oleracea, ou palmito de açaí. Por meio de inventário, foi possível verificar que a produtividade do palmito de $1^{\text {a }}$ (dap $\geq 15 \mathrm{~cm}$ ) alcançou 48,84 ind./ha e a do palmito de $2^{\text {a }}(10 \mathrm{~cm} \leq$ dap $<15 \mathrm{~cm})$, um potencial produtivo de 772,4 ind./ha.

R. Árvore, Viçosa-MG, v.29, n.5, p.719-729, 2005 
Quadro 2 - Prescrição de remoção de árvores por classe de diâmetro para Quociente de De Liocourt (q) aumentado em $50 \%(q=2,61)$ e remoção de $30 \%$ da área basal, propriedade florestal da Emapa, Município de Afuá, Pará

Table 2 - Tree removal prescriptions per diameter class according to De Liocourt value increased in 50\% $(q=2,61)$ and decreased in $30 \%$ of the basal area, Emapa Forest Enterprise, Afuá, Pará

\begin{tabular}{|c|c|c|c|c|c|c|c|c|c|c|c|}
\hline \multirow[t]{2}{*}{ Dados } & \multicolumn{10}{|c|}{ Centro de Classe Diamétrica $(\mathrm{cm})$} & \multirow{2}{*}{$\begin{array}{r}\text { Total } \\
\text { (ha) }\end{array}$} \\
\hline & 50 & 60 & 70 & 80 & 90 & 100 & 110 & 120 & 130 & $\geq 135$ & \\
\hline Observado & 22,55 & 7,79 & 3,03 & 1,66 & 0,90 & 0,55 & 0,21 & 0,21 & 0,07 & 0,07 & 37,03 \\
\hline Explorado & 5,21 & 1,14 & 0,48 & 0,68 & 0,52 & 0,41 & 0,15 & 0,19 & 0,06 & 0,07 & 8,90 \\
\hline Remanescente & 17,34 & 6,65 & 2,55 & 0,98 & 0,38 & 0,14 & 0,06 & 0,02 & 0,01 & 0,00 & 28,13 \\
\hline
\end{tabular}

\subsection{Cenários de Manejo}

Apresenta-se no Quadro 3 a proposição de remoção das espécies listadas como comerciais, para um Quociente de De Liocourt igual a 2,61 e remoção de $30 \%$ da área basal.

A importância de considerar a restrição de que pelo menos $10 \%$ das árvores de cada espécie devem permanecer em cada classe diamétrica pode ser vista no Quadro 2, na na classe de $100 \mathrm{~cm}$, verificando-se que 0,41 ind./ha pode ser removido. No entanto, o plano de colheita apresentado no Quadro 3 prescreve a remoção de 0,18 ind./ha (0,06 ind./ha para Carapa guianensis e 0,12 ind./ha para Virola surinamensis). Se o único critério de colheita fosse manter a estrutura balanceada, seriam retiradas todas as árvores dessas espécies.

Dessa aneira, as prescrições de remover 8,9 árvores quando q=2,61 e a área basal a ser explorada for 30\%, não se concretizará no plano proposto no Quadro 3 (remoção de 7,86 ind./ha). Essa diferença se deve às restrições inicialmente descritas e impostas para efetuar colheitas nessa floresta, associadas ainda ao compromisso de manter uma floresta remanescente com uma estrutura balanceada.
Quando se considerou a opção de colheita de espécies comerciais e potenciais (Cenário 2), diminuindo o conjunto de restrições à exploração florestal, devido à entrada de duas espécies potenciais (Platymiscium trinitatis e Terminalia dichotoma) no grupo das possíveis de serem exploradas, diminuiu a intensidade de remoção sobre as espécies comerciais. Tal fato é fácil de se notar pelo número de árvores que podem ser removidas de Carapa guianensis (1,37 ind./ha), Hymenaea oblongifolia (0,69 ind./ha) e Virola surinamensis (2,98 ind./ha) na classe central de $50 \mathrm{~cm}$, bem como o número dessas mesmas espécies a serem removidas na mesma classe diamétrica (Quadro 3).

O Quadro 4 ilustra o Cenário 3, no qual se consideraram as espécies comerciais, potenciais e nãocomerciais, aumentando as possibilidades de colheita da floresta. Considerando as 44 espécies arbóreas quantificadas na amostra com dap $\geq 45 \mathrm{~cm}$, apenas 13 compuseram o plano de colheita. Comparando o Quadro 4 com o Quadro 3, foi verificada uma drástica redução na intervenção sob as espécies tradicionalmente comercializadas, fato esse fácil de ser observado na classe de $50 \mathrm{~cm}$.

Quadro 3 - Plano de colheita para as espécies comerciais, considerando-se q = 2,61 e remoção de 30\% da área basal, propriedade florestal da Emapa, Município de Afuá, Pará

Table 3 - Forest management plan for trade species, considering $q=2,61$ and removal of $30 \%$ of the basal area, Emapa Forest Enterprise, Afuá, Pará

\begin{tabular}{|c|c|c|c|c|c|c|c|c|c|}
\hline \multirow[t]{2}{*}{ Espécie } & \multirow[t]{2}{*}{ Dados } & \multicolumn{7}{|c|}{ Centro de Classe Diamétrica $(\mathrm{cm})$} & \multirow{2}{*}{$\begin{array}{c}\text { Total } \\
\text { (ha) }\end{array}$} \\
\hline & & 50 & 60 & 70 & 80 & 90 & 100 & $\geq 105$ & \\
\hline \multirow[t]{2}{*}{ Carapa guianensis Aubl. } & Obs. & 1,66 & 0,69 & 0,41 & 0,21 & 0,07 & 0,07 & 0,00 & 3,11 \\
\hline & Exp. & 1,42 & 0,26 & 0,22 & 0,18 & 0,06 & 0,06 & 0,00 & 2,20 \\
\hline \multirow[t]{2}{*}{ Hymenaea oblongifolia Huber } & Obs. & 0,83 & 0,48 & 0,00 & 0,00 & 0,00 & 0,00 & 0,00 & 1,31 \\
\hline & Exp. & 0,71 & 0,18 & 0,00 & 0,00 & 0,00 & 0,00 & 0,00 & 0,89 \\
\hline \multirow[t]{2}{*}{ Virola surinamensis (Rol) Warb. } & Obs. & 3,59 & 1,86 & 0,48 & 0,55 & 0,14 & 0,14 & 0,00 & 6,76 \\
\hline & Exp. & 3,08 & 0,70 & 0,26 & 0,49 & 0,12 & 0,12 & 0,00 & 4,77 \\
\hline Total de árvores colhidas/ha & & 5,21 & 1,14 & 0,48 & 0,67 & 0,18 & 0,18 & 0,00 & 7,86 \\
\hline
\end{tabular}

R. Árvore, Viçosa-MG, v.29, n.5, p.719-729, 2005 
Quadro 4 - Plano de colheita para as espécies comerciais, potenciais e não-comerciais na várzea alta, considerando-se $\mathrm{q}=2,61$ e remoção de $30 \%$ da área basal, propriedade florestal da Emapa, Município de Afuá, Pará

Table 4 - Forest management plan for trade, potential and non-trade species, considering $q=2,61$ and removal of $30 \%$ of the basal area, Emapa Forest Enterprise, Afuá, Pará

\begin{tabular}{|c|c|c|c|c|c|c|c|c|c|}
\hline \multirow[t]{2}{*}{ Espécie } & \multirow[t]{2}{*}{ Dados } & \multicolumn{7}{|c|}{ Centro de Classe Diamétrica $(\mathrm{cm})$} & \multirow{2}{*}{$\begin{array}{l}\text { Total } \\
\text { (ha) }\end{array}$} \\
\hline & & 50 & 60 & 70 & 80 & 90 & 100 & $\geq 105$ & \\
\hline \multirow[t]{2}{*}{ Carapa guianensis Aubl. } & Obs. & 1,66 & 0,69 & 0,41 & 0,21 & 0,07 & 0,07 & 0,00 & 3,11 \\
\hline & Exp. & 0,46 & 0,11 & 0,07 & 0,10 & 0,04 & 0,06 & 0,00 & 0,84 \\
\hline \multirow[t]{2}{*}{ Hymenaea oblongifolia Huber } & Obs. & 0,83 & 0,48 & 0,00 & 0,00 & 0,00 & 0,00 & 0,00 & 1,31 \\
\hline & Exp. & 0,23 & 0,08 & 0,00 & 0,00 & 0,00 & 0,00 & 0,00 & 0,31 \\
\hline \multirow[t]{2}{*}{ Virola surinamensis (Rol) Warb. } & Obs. & 3,59 & 1,86 & 0,48 & 0,55 & 0,14 & 0,14 & 0,00 & 6,76 \\
\hline & Exp. & 0,99 & 0,30 & 0,09 & 0,26 & 0,09 & 0,12 & 0,00 & 1,85 \\
\hline \multirow[t]{2}{*}{ Platymiscium trinitatis Benth. } & Obs. & 0,21 & 0,21 & 0,21 & 0,00 & 0,00 & 0,00 & 0,00 & 0,63 \\
\hline & Exp. & 0,06 & 0,03 & 0,04 & 0,00 & 0,00 & 0,00 & 0,00 & 0,13 \\
\hline \multirow[t]{2}{*}{ Terminalia dichotoma G.F.W. Mey } & Obs. & 0,00 & 0,07 & 0,07 & 0,00 & 0,21 & 0,07 & 0,07 & 0,49 \\
\hline & Exp. & 0,00 & 0,01 & 0,01 & 0,00 & 0,13 & 0,06 & 0,06 & 0,27 \\
\hline \multirow[t]{2}{*}{ Minquartia guianensis Aubl. } & Obs. & 0,21 & 0,14 & 0,01 & 0,00 & 0,00 & 0,00 & 0,00 & 0,36 \\
\hline & Exp. & 0,06 & 0,02 & 0,01 & 0,00 & 0,00 & 0,00 & 0,00 & 0,09 \\
\hline \multirow[t]{2}{*}{ Licania macrophylla Benth } & Obs. & 2,97 & 0,83 & 0,48 & 0,00 & 0,07 & 0,00 & 0,00 & 4,35 \\
\hline & Exp. & 0,82 & 0,13 & 0,09 & 0,00 & 0,04 & 0,00 & 0,00 & 1,08 \\
\hline \multirow{2}{*}{ Inga edulis Mart. } & Obs. & 0,48 & 0,14 & 0,00 & 0,00 & 0,00 & 0,00 & 0,00 & 0,62 \\
\hline & Exp. & 0,13 & 0,02 & 0,00 & 0,00 & 0,00 & 0,00 & 0,00 & 0,15 \\
\hline \multirow[t]{2}{*}{ Eschweilera coriacea Mart. ex Berg. } & Obs. & 4,62 & 1,03 & 0,21 & 0,00 & 0,00 & 0,00 & 0,00 & 5,86 \\
\hline & Exp. & 1,28 & 0,17 & 0,04 & 0,00 & 0,00 & 0,00 & 0,00 & 1,49 \\
\hline \multirow[t]{2}{*}{ Swartizia racemosa Benth. } & Obs. & 2,97 & 1,17 & 0,28 & 0,00 & 0,00 & 0,00 & 0,00 & 4,42 \\
\hline & Exp. & 0,82 & 0,19 & 0,05 & 0,00 & 0,00 & 0,00 & 0,00 & 1,06 \\
\hline \multirow[t]{2}{*}{ Parinari excelsa Sabine } & Obs. & 0,62 & 0,21 & 0,41 & 0,62 & 0,07 & 0,21 & 0,21 & 2,35 \\
\hline & Exp. & 0,17 & 0,03 & 0,07 & 0,29 & 0,04 & 0,17 & 0,16 & 0,93 \\
\hline \multirow[t]{2}{*}{ Ceiba pentandra (L.) Gaertn. } & Obs. & 0,00 & 0,14 & 0,00 & 0,00 & 0,07 & 0,00 & 0,28 & 0,49 \\
\hline & Exp. & 0,00 & 0,02 & 0,00 & 0,00 & 0,04 & 0,00 & 0,17 & 0,23 \\
\hline \multirow[t]{2}{*}{ Anacardium giganteum Hanc. ex Engl. } & Obs. & 0,28 & 0,00 & 0,00 & 0,07 & 0,21 & 0,00 & 0,00 & 0,56 \\
\hline & Exp. & 0,08 & 0,00 & 0,00 & 0,03 & 0,13 & 0,00 & 0,00 & 0,24 \\
\hline Total de árvores colhidas / ha & & 5,10 & 1,11 & 0,47 & 0,68 & 0,51 & 0,41 & 0,39 & 8,67 \\
\hline
\end{tabular}

A prescrição de remover 8,9 ind./ha (Quadro 2) para um q $=2,61$ e remoção de $30 \%$ da área basal é agora próxima àquela obtida no Quadro 4 (8,67 ind./ha). Essa é uma possibilidade que amplia o leque de exploração para 10 outras espécies, além das três espécies comerciais existentes na área. Esse aumento do rol das espécies a serem exploradas dentro dos limites que a floresta pode diminuirá a pressão de exploração sobre poucas espécies, já que este último fato geralmente compromete a produção sustentável.

\subsection{Valoração dos Planos de Colheita}

A valoração foi realizada com base no produto do número médio de toras por classe de tora de cada espécie, com número de árvores exploradas por hectare e respectivos preços. Aos valores da madeira em pé a ser explorada foi incluído o rendimento da exploração do palmito de açaí, gerando, assim, a valoração total por hectare dos planos de colheita. Apesar de esses produtos apresentarem ciclos de corte diferentes, não são atividades antagônicas, portanto podem ser executadas na mesma época.

Comparando a opção que abrange as espécies comerciais + potenciais (US\$ 445,90/ha) em relação à opção que contempla só as espécies comerciais (US\$ 427,80/ha), houve um acréscimo na renda de US\$ $18,1 /$ ha, ou 4,23\%. Quando a opção pelo cenário que considera também as espécies potenciais e as nãocomerciais (US\$ 424,40/ha), há uma redução da renda em relação ao cenário que considera só as comerciais $(0,79 \%)$.

Esse fato ocorreu porque entraram muitas espécies não-comerciais no plano de colheita, as quais têm preço de venda mais baixo, gerando, conseqüentemente, menor renda. Portanto, foi possível verificar que, apesar de

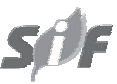

R. Árvore, Viçosa-MG, v.29, n.5, p.719-729, 2005 
o plano de colheita que considera a exploração das comerciais + potenciais + não-comerciais diminuir sensivelmente $(61,8 \%)$ a pressão sobre as espécies comerciais, o plano de colheita que gera o maior lucro é aquele que leva em conta as espécies comerciais + potenciais.

O aproveitamento do palmito (US\$94,00/ha) é uma alternativa para se agregar valor à produção florestal. A participação desse produto em cada um dos cenários de manejo considerados neste estudo foi de 22 (Cenário 1), 21 (Cenário 2) e 22\% (Cenário 3) da renda total.

Foi possível observar que o processamento da madeira advinda de cada um dos três cenários de manejo poderá gerar um lucro por hectare de US\$ 3.925,80, US\$3.945,40 e US\$2.156,40, o que representa um aumento de receita da ordem de 917,$7 ; 884,8$ e 508,1\%, nos Cenários 1,2 e 3 , respectivamente. Tal fato reforça a importância de se agregar valor aos produtos extraídos da floresta.

\subsection{Potencial de Exploração das Espécies}

No Quadro 5 são apresentados os índices que caracterizam a regeneração natural das espécies que compuseram os três cenários de manejo adotados, oriundos do estudo de Gama et al. (2003), realizado nas mesmas parcelas e período de estudo, para melhor inferir sobre as espécies selecionadas nos planos de manejo.

Entre as espécies comerciais, observou-se que Virola surinamensis apresentou densidade absoluta de regeneração natural muito alta, com 10.348,28 ind./ha. No entanto, 99,57\% das árvores ocorreram na classe de tamanho (CT1) com até 1,5 m de altura; $0,27 \%$ na classe de tamanho (CT2), com altura entre 1,5 e $3,0 \mathrm{~m} ; 0,10 \%$ na classe de tamanho (CT3) entre $3 \mathrm{~m}$ de altura e $5 \mathrm{~cm}$ de dap; $0,03 \%$ na classe de tamanho (CT4) entre 5 e $10 \mathrm{~cm}$ de dap; e $0,03 \%$ na classe de tamanho (CT5) entre 10 e $15 \mathrm{~cm}$ de dap. Essa espécie apresentou alto potencial de regeneração natural em relação às outras espécies comerciais, que também fizeram parte do plano de colheita. Pode-se inferir que um manejo para a condução da regeneração natural é crucial para favorecer o desenvolvimento dessa espécie, pois, embora existam espécies potenciais e não-comerciais que possam substituí-la, nenhuma possui a mesma combinação das características tecnológicas de sua madeira, rápido crescimento, excelente forma de fuste e abundância local.

Quadro 5 - Potencial de regeneração natural das espécies comerciais, potenciais e não- comerciais. Propriedade florestal da Emapa, Município de Afuá, Pará

Table 5 - Potential for natural regeneration of trade, potential and non-trade species, Emapa Forest Enterprise, Afuá, Pará

\begin{tabular}{|c|c|c|c|c|c|}
\hline$\#$ & Cenário/Espécie & DARN & FARN & $\mathrm{CT}$ & $\begin{array}{c}\text { Potencial da } \\
\text { RegeneraçãoNatural }\end{array}$ \\
\hline & Comerciais & & & & \\
\hline 1 & Virola surinamensis & $10.348,28$ & 100 & $1,2, \ldots, 5$ & MA \\
\hline 2 & Carapa guianensis & 65,52 & 34 & 1,3 e 5 & M \\
\hline 3 & Hymenaea oblongifolia & 37,93 & 21 & 1 e 3 & B \\
\hline 4 & $\begin{array}{l}\text { Potenciais } \\
\text { Platymiscium trinitatis }\end{array}$ & 20,69 & 14 & 1 e 3 & B \\
\hline 5 & Terminalia dichotoma & 31,03 & 14 & 1 & B \\
\hline 6 & $\begin{array}{l}\text { Não-comerciais } \\
\text { Eschweilera coriacea }\end{array}$ & 258,62 & 79 & $1,2, \ldots, 5$ & A \\
\hline 7 & Swartizia racemosa & 186,21 & 72 & $1,2,3$ e 5 & A \\
\hline 8 & Licania macrophylla & 289,66 & 41 & 1 e 3 & M \\
\hline 9 & Parinari excelsa & 68,97 & 41 & 1 e 2 & M \\
\hline 10 & Inga edulis & 644,83 & 90 & $1,2, \ldots, 5$ & MA \\
\hline 11 & Anacardium giganteum & 117,24 & 10 & 1 & M \\
\hline 12 & Minquartia guianensis & 17,24 & 14 & 1 e 2 & MB \\
\hline 13 & Ceiba pentandra & 0,00 & - & - & - \\
\hline
\end{tabular}

Em que: $\mathrm{DARN}=$ densidade absoluta da regeneração natural por hectare; FARN = freqüência absoluta da regeneração natural; CT = ocorrência nas classes de tamanho (CT1 - 0,3 $\mathrm{m} \leq \mathrm{h}<1,5 \mathrm{~m}, \mathrm{CT} 2-1,5 \mathrm{~m} \leq \mathrm{h}<3,0 \mathrm{~m}, \mathrm{CT} 3-\mathrm{h} \geq 3,0 \mathrm{~m}$ a dap $<5,0 \mathrm{~cm}, \mathrm{CT} 4-5,0 \mathrm{~m} \leq$ dap $<10 \mathrm{~cm}$ e CT5 $-10,0 \mathrm{~m} \leq$ dap $<15 \mathrm{~cm}) ; \mathrm{MA}=$ muito alta, $\mathrm{A}=$ alta, $\mathrm{M}=$ média, $\mathrm{B}=$ baixa; e $\mathrm{MB}=$ muito baixa.

Where: DARN = absolute density of natural regeneration per hectare; FARN = absolute frequency of natural regeneration; $P C T=$ presence in the size (CT1 - $0,3 \mathrm{~m} \leq h<1,5 \mathrm{~m}, \mathrm{CT} 2-1,5 \mathrm{~m} \leq \mathrm{h}<3,0 \mathrm{~m}, \mathrm{CT} 3-\mathrm{h} \geq 3,0 \mathrm{~m}$ to $\mathrm{DBH}<5,0 \mathrm{~cm}, \mathrm{CT} 4-5,0 \mathrm{~m} \leq \mathrm{DBH}<10 \mathrm{~cm}$ and CT5 $-10,0 \mathrm{~m} \leq \mathrm{DBH}<15 \mathrm{~cm}) ; M A=$ too high, $A=$ high, $M=$ medium, $B=$ low, $M B=$ very low.

R. Árvore, Viçosa-MG, v.29, n.5, p.719-729, 2005 
Deve-se incentivar o recrutamento da regeneração natural para as maiores classes de tamanho, por meio de tratamento silvicultural (refinamento e liberação), e, desse modo, aumentar os estoques de crescimento e exploração. Outro ponto que representa maior chance de obter sucesso com essa prática é que a distribuição espacial da regeneração natural de Virola surinamensis foi detectada em toda a área amostrada, como se pode observar ao analisar a freqüência absoluta, que foi de 100\% (Quadro 5).

Carapa guianensis, Hymenaea oblongifolia e as espécies potenciais (Terminalia dichotoma e Platymiscium trinitatis) apresentaram baixa densidade relativa na regeneração natural. Os indivíduos dessas espécies encontraram-se quase que exclusivamente na primeira classe de regeneração natural, apresentam distribuição espacial agregada - conforme o valor de suas freqüências absolutas. Também para essas espécies, recomendam-se os mesmos tratamentos silviculturais indicados para a virola, devido ao fato de eles participarem do mesmo grupo ecológico. Segundo Gama et al. (2003), são espécies-clímax exigentes de luz.

Vale destacar que as espécies potenciais, apesar de apresentarem números abaixo do desejável na regeneração natural e no estoque de exploração, têm uma característica interessante, que é o alto número de toras por árvore, apresentando ainda fuste retilíneo, o que as torna mais aproveitáveis. Nesse quesito, essas duas espécies superaram a Virola surinamensis.

Entre as espécies não-comerciais, podem-se destacar: Inga edulis, que apresentou alta densidade (644,83 ind./ha), distribuição por quase toda a área e ocorrência em todas as classes de tamanho da regeneração natural; e Eschweilera coriacea e Swartizia racemosa, que também apresentaram boa distribuição nas diversas classes de tamanho da regeneração natural, no entanto exibiram um número pequeno de toras de $4 \mathrm{~m}$ por árvore, em comparação com as espécies comerciais, embora retilíneas.

Dentre as espécies com uma densidade ainda superior àquela encontrada nas espécies comerciais (exceção da Virola surinamensis), mas com uma distribuição em torno de $40 \%$ na área, podem-se citar Licania macrophylla e Parinari excelsa, no entanto estas se encontram quase que totalmente na classe de tamanho que compreende árvores com altura inferior a 1,5 m. Entre as espécies que apresentaram uma distribuição espacial extremamente agregada, encontraram-se: Anacardium giganteum (117,24 ind./ ha) e Minquartia guianensis (17,24 ind./ha). Ceiba pentandra não foi identificada como integrante do estoque de regeneração.

\section{CONCLUSÃO}

Com base nos dados analisados para a condição de floresta de várzea não explorada no estuário amazônico, concluiu-se que:

- As espécies comerciais fitossociologicamente mais importantes foram: Virola surinamensis, Carapa guianensis e Hymenaea oblongifolia. Entre as espécies potenciais, destacou-se Terminalia dichotoma, enquanto no grupo das espécies não-comerciais, Eschweilera coriacea, Swartizia racemosa e Licania macrophylla.

- Para que a floresta permaneça balanceada após a colheita, o Quociente de De Liocourt (q) tem que ser maior que o original, sendo as melhores opções de colheita: $q=2,61$, com remoção de $25 \%$ da área basal; e as opções de $q=2,61$ e q=3,48, com remoção de $30 \%$ da área basal.

- O cenário de manejo que contempla as espécies comerciais e as potenciais é o que gera maior lucro. Adotando o plano de colheita que utiliza um Quociente de De Liocourt $50 \%$ maior do que o original $(q=2,61)$ e remoção de $30 \%$ da área basal, podem-se obter lucros de US\$ 445,90/ha com a madeira em pé, juntamente com a colheita do palmito, e de US\$3.945,40/ha após o desdobro.

- Ao utilizar o conceito de floresta balanceada, juntamente com as restrições que garantem a preservação de espécies raras, daquelas de menor porte, das árvores porta-sementes e das espécies proibidas de corte, dentre outras previamente descritas, pode-se chegar a práticas de manejo comprometidas com a produção sustentável e com a manutenção da diversidade genética da flora e fauna, dentro de limites ambientalmente aceitáveis.

\section{REFERÊNCIAS BIBLIOGRÁFICAS}

ARAÚJO, A. P.; JORDY FILHO, S.; FONSECA, W. N. A vegetação da Amazônia brasileira. In: SIMPÓSIO DO TRÓPICO ÚMIDO, 1., 1984, Belém. Anais... Belém: EMBRAPA-CPATU, 1986. p. 135152. (EMBRAPA-CPATU. Documentos, 36).

R. Árvore, Viçosa-MG, v.29, n.5, p.719-729, 2005 
BARRETO, P. et al. Costs and benefits of Forest management for timber production in eastern Amazônia. Forest Ecology and

Management, v.108, p.9-26, 1998.

BARROS, P. L. C. et al. Novos coeficientes para converter volume geométrico ou volume francon para madeira serrada a serem usados pelo Ibama (PA). Belém: FCAP. Serviço de Documentação e Informação, 2002. p.43-57. (FCAP. Informe Técnico, 28)

BERTAULT, J. G.; DUPUY, B.; MAITRE, H. F. La silvicultura para la ordenación sostenible del bosque tropical húmedo. Unassylva, v. 46, n. 181, p. $3-9,1995$.

BUSCHBACHER, R. J. Natural forest managemesnt in the humid tropic: ecological, social, and economic considerations. Ambio, v. 19, n. 5, p. 253-258, 1990.

CAMPOS, J. C. C.; RIBEIRO, J. C.; COUTO, L. Emprego da distribuição diamétrica na determinação de corte de matas naturais submetidas ao sistema de seleção. Revista Árvore, v.7, n.2, p.110-122, 1983.

CRONQUIST, A. The evolution and classification of flowering plants. New York: The New York Botanical Garden, 1988. 555 p.

CURTIS, J. T.; MCINTOSH, R. P. An upland forest continuum in the prairie forest border region of Wisconsin. Ecology, v.32, n.3, p. 476496, 1951

FAO. Manual de inventario florestal con especial referencia a los bosques mistos tropicales. Roma: 1974. 195 p.

FINEGAN, B. et al. Los bosques húmedos tropicales de América Central: su manejo sostenible es posible. Revista Forestal Centroamericana, v.2, n.6, p.17-27, 1993.

FINOL, U. H. Nuevos parametros a considerarse en el analises estructural de las Selvas Virgines tropicais. Revista Florestal Venezolana, v.14, n.21, p.29-42, 1971.

R. Árvore, Viçosa-MG, v.29, n.5, p.719-729, 2005
GAMA, J.R.V. et al. Estrutura e potencial futuro de utilização da regeneração natural de floresta de várzea alta mo município de Afuá, Estado do Pará. Ciência Florestal, v.12, n.13, p.71-82, 2003.

BRASIL. Ministério da Agricultura. Instituto Brasileiro do Meio Ambiente e dos Recursos Naturais Renováveis - IBAMA. Portaria no 48 de 10 de julho de 1995. Brasília: 1995. 41 p.

JARDIM, F. C. S.; HOSOKAWA, R. T. Estrutura da floresta equatorial úmida da Estação Experimental de Silvicultura Tropical do INPA Acta Amazonica, v. 16/17, p. 411-508, 1986/87.

MACEDO, D. S. M. S. Estrutura e manejo de uma floresta de várzea do estuário amazônico. 1996. 117f. Dissertação (Mestrado em Ciências Florestais) - Escola Superior de Agricultura Luiz de Queiroz, Piracicaba, 1996.

MINETTI, L.J. et al. Análise técnica e econômica do corte florestal planejado de floresta tropical úmida de terra-firme na Amazônia ocidental.

Revista Árvore, v.24, n.4, p.429-435, 2000.

RAMOS, C.A.P.. Possibilidades de otimização do uso florestal para pequenos produtores nas várzeas amazônicas: um estudo na costa amapaense. 2000. 112f. Dissertação (Mestrado em Ciências Florestais) - Faculdade de Ciências Agrárias do Pará, Belém, 2000.

RIBEIRO, R.N.S.; TOURINHO, M.M.; SANTANA, A.C. Avaliação da sustentabilidade agroambiental de unidades produtivas agroflorestais em várzeas flúvio marinhas de Cametá - Pará. . Acta

Amazonica, v.34, n.3, p. 359 - 374, 2004.

SCOLFORO, J. R. S. SISNAT - sistema de manejo para florestas nativas. Lavras: UFLA/FAEPE, 1998. 25p.

SCOLFORO, J. R. S.; MELLO, J. M. Inventário florestal. Lavras: UFLA/FAEPE, 1997. 344 p.

SILVA, J. N. M. The behaviour of the tropical rain forest of the Brazilian Amazon after logging. 1989. 325f. Thesis (Doctorate in Forestry Science) - University of Oxford, Oxford, 1989. 
SILVA, J. N. M. et al. Equações de volume para a Floresta Nacional do Tapajós. Boletim de Pesquisa Florestal, v.8/9, p. 50-63, 1984.

SILVA, J.N.M. et al. Growth and yield of a tropical rain forest in the Brazilian Amazon 13 years after logging. Forest Ecology and

Management, v.71, p.267-274, 1995.

SUDAM/Projeto de Hidrologia e Climatologia da Amazônia. Atlas Climatológico da Amazônia Brasileira. Belém: 1984. 125p.
UHL, C.; VIEIRA, I.C.G. Ecological impacts of selective logging in the Brazilian Amazon: a case study from the Paragominas region in the state of Para.. Biotropica, n.21, p.98-106, 1989.

VIDAL, E.; VIANA, V. M.; BATISTA, J. L. F. Crescimento de floresta tropical três anos após colheita de madeira com e sem manejo florestal na Amazônia oriental. Scientia Forestalis, n. 61, p. 133-143, 2002.

VIEIRA, L. S. Manual da Ciência do Solo: com ênfase aos solos tropicais. São Paulo: Agronômica Ceres, 1988.464p. 